\title{
Inequalities in the burden of female breast cancer in Brazil, 1990-2017
}

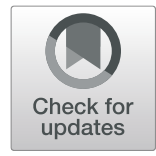

\author{
Maximiliano Ribeiro Guerra ${ }^{1 *}$ (D, Mário Círio Nogueira ${ }^{1}$, Deborah Carvalho Malta ${ }^{2}$, Camila Soares Lima Côrrea', \\ Maria de Fatima Marinho de Souza ${ }^{3}$, Maria Paula Curado ${ }^{4}$, Mariana Santos Felisbino-Mendes ${ }^{2}$, Meghan Mooney ${ }^{5}$, \\ Mohsen Naghavi ${ }^{5}$ and Maria Teresa Bustamante-Teixeira ${ }^{1}$
}

\begin{abstract}
Background: Breast cancer is the most frequently diagnosed cancer in women and the leading cause of cancer death among females worldwide. In recent decades, breast cancer death rates have been stable or decreasing in more developed regions; however, this has not been observed in less developed regions. This study aims to evaluate inequalities in the burden of female breast cancer in Brazil including an analysis of interregional and interstate patterns in incidence, mortality and disability-adjusted life years (DALYs) rates from 1990 to 2017, and mortality-to-incidence ratio (MIR), and their association with the Socio-demographic Index (SDI).
\end{abstract}

Methods: Using estimates from the global burden of disease (GBD) study, we applied a spatial exploratory analysis technique to obtain measurements of global and local spatial correlation. Percentage changes of breast cancer incidence, mortality, and DALYs rates between 1990 and 2017 were calculated, and maps were developed to show the spatial distribution of the variables. Spatial panel models were adjusted to investigate the association between rates and SDI in Brazilian states.

Results: In Brazil, while breast cancer mortality rate have had modest reduction $(-4.45 \%$; $95 \%$ UI: $-6.97 ;-1.76)$ between 1990 and 2017, the incidence rate increased substantially (+39.99\%; 95\% UI: 34.90; 45.39). Breast cancer incidence and mortality rates in 1990 and 2017 were higher in regions with higher SDI, i.e., the most developed ones. While SDI increased in all Brazilian states between 1990 and 2017, notably in less developed regions, MIR decreased, more notably in more developed regions. The SDI had a positive association with incidence rate and a negative association with MIR.

Conclusion: Such findings suggest an improvement in breast cancer survival in the period, which may be related to a broader access to diagnostic methods and treatment. This study also revealed the inequality in breast cancer outcomes among Brazilian states and may guide public policy priorities for disease control in the country.

Keywords: Breast neoplasm, Incidence, Mortality, Global burden

\footnotetext{
* Correspondence: guerramr@hotmail.com

${ }^{1}$ Graduate Program in Public Health, Universidade Federal de Juiz de Fora (UFJF), Juiz de Fora, Minas Gerais, Brazil

Full list of author information is available at the end of the article
} 


\section{Background}

Breast cancer is the most common type of cancer and the main cause of cancer-related death among females worldwide [1-3], as well as the leading cause of disability-adjusted life years (DALYs) due to cancer in women [3]. Although breast cancer incidence rates are higher in more developed regions, the incidence in less developed regions has increased in the last decades, while it has remained relatively stable or even reduced in more developed regions [3, 4]. Likewise, breast cancer death rates have been decreasing in many high-income countries, while mortality rates continue to increase in several low- and middle-income countries, such as those in Latin America and the Caribbean, and parts of Asia [4].

In general, the range in mortality rates between world regions is lower than for incidence rates as a result of better survival of breast cancer in developed regions. The use of the mortality-to-incidence ratio (MIR), in this context, can allow a better identification of the heterogeneity in breast cancer burden [5].

Nevertheless, even within regions, there may be remarkable geographic variability in disease incidence and mortality patterns. In Central and South America, for instance, there was a wide variation in breast cancer rates across the region, with the highest rates in Central America almost 50\% lower than the highest rates observed in South America. Breast cancer incidence in Argentina, Brazil, and Uruguay, for instance, reached levels similar to those of other countries of very high economic development, such as the USA and Canada. On the other hand, while Uruguay and Argentina had some of the highest breast cancer mortality rates in the world, Guatemala and Nicaragua had the lowest rates [6].

In Brazil, breast cancer incidence increased from an age-standardized rate of 54.73 (95\% U.I.: 45.52-60.30) in 1990 to 74.02 (95\% U.I.: 61.90-85.86) in 2015 [7]. While breast cancer incidence has increased in Brazil, there have been conflicting reports as to if breast cancer mortality has remained stable [7-9] or increased [10-14]. However, all studies have shown regional disparities in breast cancer mortality in the Brazilian regions, usually with rates remaining stable or declining in more developed regions and increasing in less developed regions [6, $8,10,11,15,16]$.

Regional variation in breast cancer rates over time most likely reflects changes and differences in multiple factors including the availability of early detection and healthcare access, the prevalence of physical inactivity, alcohol consumption and excess weight, and variability in registration practices [2, 4, 6, 17]. Given these known factors that contribute to the global burden of breast cancer, studies that are assessing breast cancer in a given region over time should always consider the possibility of intra and interregional variation, especially in regions with limited health resources [18]. In addition, the use of maps to identify the areas of greater risk, taking into account the disease control network, can be used to outline strategies to reduce the magnitude of breast cancer on the local population.

This study aims to evaluate inequalities in the burden of breast cancer in Brazil by means of the analysis of interregional and interstate variation in incidence, mortality and DALYs rates from 1990 to 2017, and MIR, and their association with the sociodemographic index (SDI).

\section{Methods}

\section{Data sources}

All analyses were carried out using data of global burden of disease study 2017 (GBD 2017), coordinated by the Institute for Health Metrics and Evaluation (IHME) that estimated the burden of diseases, injuries, and risk factors for 195 countries and territories and at the subnational level for a subset of countries [19].

Input data to estimate breast cancer rates came from the Brazilian cancer registry for incidence data, and from the mortality information system of the Brazilian Ministry of Health for death data, with adjustment for underreporting of deaths, and for ill-defined/nonspecific causes, called garbage codes [20]. We used the IBGE (Brazilian Institute of Geography and Statistics) codes to show the spatial configuration of the Brazilian regions and states (Figure S1).

\section{Variables of interest}

The outcome variables were rates of age-standardized breast cancer death (ASDR), incidence (ASIR) and disability-adjusted life year (DALYs) per 100,000 women, and mortality-to-incidence ratio (MIR). We reported 95\% uncertainty intervals (95\% UI) for mortality, incidence and DALYs rates, and respective percentual changes between 1990 and 2017 years.

The percentual changes were calculated as the proportional difference between rates from the year 2017 and the year 1990 in relation to the year 1990. In the GBD study methods, the uncertainty intervals are estimated systematically using Monte Carlo approach with the repetition of 1000 draws for each estimate. Thus, the 95\% UIs are the 2.5th and 97.5th percentiles of the draw-level values are the lower and upper bound, respectively [21].

We divided publicly available data of all agestandardized death rates by all incidence rates of breast cancer in females from the GBD 2017 study to calculate the mortality-to-incidence ratio (MIR) [22].

The exposure variable was the socio-demographic index (SDI). Developed by GBD researchers, the socio- 
demographic index (SDI) is a measure of sociodemographic development of a geographic region, based on average income per person, educational attainment, and total fertility rate. It contains an interpretable scale in which zero (0) represents the lowest sociodemographic development observed across all GBD geographies from 1970 to 2017, and one (1) represents the opposite, i.e., the highest SDI for the same period [23].

All estimates were made for Brazil and states (i.e., the Federative Units - FU) from 1990 to 2017. All variables were grouped into quintiles in order to visualize its spatial distribution in Brazilian states based on its value for years 1990 and 2017. All estimates and their respective percentage variation were shown in tables and maps. Temporal distribution plots of indicators over the considered period were made for Brazil and states.

\section{Statistical analyses}

For exploratory analysis, scatter plots were used, and Pearson correlation coefficients between the variables were estimated. We estimated a measure of global spatial autocorrelation, the Moran I coefficient, which evaluates whether the measure of the variable in one region is correlated with the measure of the same variable in neighboring regions. We also estimated a local spatial correlation, the LISA estimator [24], and from its results were created maps of local clusters, that is, groups of regions with values for a variable significantly similar to their neighbors. The clusters are called high-high and low-low, respectively for the cluster of values above and below the average of all regions. In order to elaborate the spatial weights, the criterion of four nearest neighbors was used, after estimations with several options to verify which neighborhood matrix captured more the spatial dependence.

To investigate the relationship between SDI and the other variables in Brazilian states from 1990 to 2017, panel data models were adjusted. The Hausman test [25] was used to guide the choice between fixed or randomeffects model, and the Pesaran test [26] to evaluate the presence of cross-sectional dependence in observations, which would indicate the need to adjust for the spatial correlation by spatial panel models. Spatial models known as SAR (spatial auto-regressive) include a spatial lag of the dependent variable as covariate.

All analyzes were performed in the $\mathrm{R}$ statistical program [27], using the suggestions of packages and functions for spatial analysis from Anselin [28].

\section{Results}

For Brazil as a whole, breast cancer mortality rates ranged from 15.28 (95\% UI: $14.95 ; 15.58)$ in 1990 to 14.60 (95\% UI: 14.23 ; 14.97$)$, with a decrease of $4.45 \%$
(95\% UI: -6.97; -1.76) between 1990 and 2017 years. However, there was a geographical variation in mortality rates. Although in most states, death rates were stable in the period, some states had an increase in mortality, mainly in the Northeast region, with the largest increases in the states of Rio Grande do Norte $(+27.56 \%$; 95\% UI: $13.04 ; 44.00)$ and Alagoas (+28.97\%; 95\% UI: $12.37 ; 47.11)$. Nearly half of the states in the South, Southeast, and Central-West regions had a reduction in mortality rates in the period, with the largest reductions in the states of Rio Grande do Sul (-14.08\%; 95\% UI: -20.22 ; -7.54), São Paulo (-14.26\%; 95\% UI: -19.84 ; -8.73 ), and Distrito Federal (-14.71\%; 95\% UI: -22.66 ; -5.40) (Table 1; Figs. 1 and S2).

Breast cancer incidence rates for Brazil in 1990 and 2017 were 29.75 (95\% UI: 29.00; 30.48), and 41.65 (95\% UI: 40.22; 43.13) new cases per 100,000 women, respectively, with an increase of $39.99 \%$ (95\% UI: 34.90; 45.39) between 1990 and 2017 years. As well as for mortality, incidence rates were also higher in the Southeast and South regions, lower in the North and Northeast regions, and intermediate in the CentralWest region. All Brazilian states showed an increase in incidence rate from 1990 to 2017, with a higher increase for some states of the North (Amazonas) and Northeast (Rio Grande do Norte, Alagoas, Sergipe, and Bahia) regions, which had lower values initially (Table 1; Figs. 2 and S2).

The MIR decreased in all states, more notably in the South and Southeast regions, in which it reached the lowest values in 2017 (Table 2; Figs. 3 and S3).

Taking into account the Brazilian regions, SDI had higher values in the Southeast, South, and Central West, and lower values in the North and Northeast. From 1990 to 2017, SDI exhibited a steady increase in all states, although this increase was higher for states of the North and Northeast regions, which had the lowest values in 1990 (Table 2; Figs. 4 and S3).

The DALYs rates had similar behavior to mortality, not only with higher values in the Southeast, South, and Central-West regions, but also with the greatest reductions in these regions between 1990 and 2017 years. However, only three states had a reduction in the period: Distrito Federal (-17.73\%; 95\% UI: -25.29 ; -8.90$)$, Rio Grande do Sul $(-12.23 \%$; 95\% UI: $-18.94 ;-5.03)$, and São Paulo (-11.73\%; 95\% UI: $-17.90 ;-5.42$ ) (Table 1; Figs. 5 and S4).

In the exploratory analyzes, there was a significant correlation between all the variables both in 1990 and 2017 years. The SDI had a positive correlation with ASDR, ASIR, and DALYs, and negative correlation with MIR in 1990 and 2017 years (Table 3). Almost all the indicators exhibited significant spatial autocorrelation, except for MIR in 1990 (Table 4). 
Table 1 Age-standardized female breast cancer death (ASDR), incidence (ASIR) and disability-adjusted life years (DALYs) rates according to Brazilian states and regions in 1990 and 2017, and percentage change $(\Delta \%)$

\begin{tabular}{|c|c|c|c|c|c|c|c|c|c|c|}
\hline \multirow[b]{2}{*}{$\begin{array}{l}\text { IBGE } \\
\text { CODE }\end{array}$} & \multirow[b]{2}{*}{ REGION/UF } & \multicolumn{3}{|l|}{$\begin{array}{l}\text { ASDR } \\
\text { (UI 95\%) }\end{array}$} & \multicolumn{3}{|c|}{$\begin{array}{c}\text { ASIR } \\
(\mathrm{UI} 95 \%)\end{array}$} & \multicolumn{3}{|l|}{$\begin{array}{l}\text { DALY } \\
\text { (UI 95\%) }\end{array}$} \\
\hline & & 1990 & 2017 & $\Delta \%$ & 1990 & 2017 & $\Delta \%$ & 1990 & 2017 & $\Delta \%$ \\
\hline \multirow[t]{2}{*}{-} & \multirow[t]{2}{*}{ Brazil } & 15.28 & 14.60 & -4.45 & 29.75 & 41.65 & 39.99 & 440.44 & 428.37 & -2.74 \\
\hline & & $14.95-15.58$ & $\begin{array}{l}\text { 14.23; } \\
14.97\end{array}$ & $\begin{array}{l}-6.97 \\
-1.76\end{array}$ & $\begin{array}{l}\text { 29.00; } \\
30.48\end{array}$ & $\begin{array}{l}40.22 ; \\
43.13\end{array}$ & $\begin{array}{l}34.90 \\
45.39\end{array}$ & $\begin{array}{l}430.03 \\
450.96\end{array}$ & $\begin{array}{l}415.39 ; \\
442.43\end{array}$ & $-5.72 ; 0.44$ \\
\hline 1 & \multirow{3}{*}{$\begin{array}{l}\text { North region } \\
\text { Rondônia }\end{array}$} & & & & & & & & & \\
\hline \multirow[t]{2}{*}{11} & & 10.05 & 10.51 & 4.62 & 17.90 & 28.19 & 57.55 & 294.76 & 307.46 & 4.31 \\
\hline & & $9.01 ; 11.11$ & $\begin{array}{l}9.01 \\
12.17\end{array}$ & $\begin{array}{l}-12.02 \\
24.77\end{array}$ & $\begin{array}{l}15.83 ; \\
20.01\end{array}$ & $\begin{array}{l}\text { 23.91; } \\
32.90\end{array}$ & $\begin{array}{l}29.80 \\
89.22\end{array}$ & $\begin{array}{l}263.37 \\
329.48\end{array}$ & $\begin{array}{l}260.63 \\
357.79\end{array}$ & $\begin{array}{l}-13.79 \\
24.66\end{array}$ \\
\hline \multirow[t]{2}{*}{12} & \multirow[t]{2}{*}{ Acre } & 8.59 & 9.14 & 6.34 & 15.30 & 21.90 & 43.11 & 256.05 & 261.43 & 2.10 \\
\hline & & $7.98 ; 9.25$ & $8.36 ; 9.95$ & $\begin{array}{l}-5.58 ; \\
18.96\end{array}$ & $\begin{array}{l}14.10 \\
16.68\end{array}$ & $\begin{array}{l}19.68 ; \\
24.16\end{array}$ & $\begin{array}{l}24.76 \\
63.17\end{array}$ & $\begin{array}{l}236.28 \\
277.19\end{array}$ & $\begin{array}{l}237.09 ; \\
285.87\end{array}$ & $\begin{array}{l}-10.34 \\
15.60\end{array}$ \\
\hline \multirow[t]{2}{*}{13} & \multirow[t]{2}{*}{ Amazonas } & 9.69 & 11.60 & 19.64 & 17.80 & 30.39 & 70.77 & 287.14 & 345.43 & 20.30 \\
\hline & & $8.87 ; 10.52$ & $\begin{array}{l}10.67 \\
12.50\end{array}$ & $7.02 ; 34.76$ & $\begin{array}{l}16.18 \\
19.45\end{array}$ & $\begin{array}{l}27.45 \\
33.49\end{array}$ & $\begin{array}{l}49.70 \\
95.09\end{array}$ & $\begin{array}{l}261.73 \\
313.59\end{array}$ & $\begin{array}{l}317.05 \\
375.15\end{array}$ & $6.76 ; 36.09$ \\
\hline \multirow[t]{2}{*}{14} & \multirow[t]{2}{*}{ Roraima } & 12.14 & 11.57 & -4.68 & 21.32 & 28.27 & 32.64 & 328.78 & 305.37 & -7.12 \\
\hline & & $10.69 ; 13.73$ & $\begin{array}{l}9.69 ; \\
13.50\end{array}$ & $\begin{array}{l}-23.57 \\
17.98\end{array}$ & $\begin{array}{l}18.55 ; \\
24.43\end{array}$ & $\begin{array}{l}23.48 ; \\
33.40\end{array}$ & $5.12 ; 68.19$ & $\begin{array}{l}283.97 \\
373.73\end{array}$ & $\begin{array}{l}253.42 \\
360.18\end{array}$ & $\begin{array}{l}-26.34 \\
16.70\end{array}$ \\
\hline \multirow[t]{2}{*}{15} & \multirow[t]{2}{*}{ Pará } & 10.03 & 10.31 & 2.73 & 18.55 & 25.53 & 37.64 & 303.22 & 304.74 & 0.50 \\
\hline & & $9.28 ; 10.83$ & $\begin{array}{l}9.52 \\
11.10\end{array}$ & $\begin{array}{l}-7.63 \\
14.29\end{array}$ & $\begin{array}{l}16.82 ; \\
20.32\end{array}$ & $\begin{array}{l}23.16 ; \\
28.03\end{array}$ & $\begin{array}{l}21.70 \\
56.00\end{array}$ & $\begin{array}{l}278.48 \\
328.75\end{array}$ & $\begin{array}{l}279.40 \\
328.33\end{array}$ & $\begin{array}{l}-10.33 \\
12.58\end{array}$ \\
\hline \multirow[t]{2}{*}{16} & \multirow[t]{2}{*}{ Amapá } & 8.63 & 9.59 & 11.13 & 16.76 & 24.57 & 46.58 & 252.75 & 277.91 & 9.95 \\
\hline & & $7.94 ; 9.34$ & $\begin{array}{l}8.77 \\
10.42\end{array}$ & $\begin{array}{l}-0.78 \\
24.82\end{array}$ & $\begin{array}{l}15.24 \\
18.39\end{array}$ & $\begin{array}{l}22.30 ; \\
27.15\end{array}$ & $\begin{array}{l}28.55 \\
67.05\end{array}$ & $\begin{array}{l}231.80 \\
275.31\end{array}$ & $\begin{array}{l}253.65 \\
304.13\end{array}$ & $\begin{array}{l}-2.76 \\
24.22\end{array}$ \\
\hline \multirow[t]{2}{*}{17} & \multirow[t]{2}{*}{ Tocantins } & 9.60 & 10.06 & 4.83 & 18.43 & 26.78 & 45.26 & 269.92 & 299.59 & 10.99 \\
\hline & & $8.21 ; 11.01$ & $\begin{array}{l}9.10 \\
11.03\end{array}$ & $\begin{array}{l}-11.76 \\
25.29\end{array}$ & $\begin{array}{l}15.23 ; \\
21.40\end{array}$ & $\begin{array}{l}23.67 ; \\
29.98\end{array}$ & $\begin{array}{l}20.24 \\
77.49\end{array}$ & $\begin{array}{l}222.17 \\
316.32\end{array}$ & $\begin{array}{l}269.37 \\
330.84\end{array}$ & $\begin{array}{l}-8.12 ; \\
35.35\end{array}$ \\
\hline 2 & Northeast region & & & & & & & & & \\
\hline \multirow[t]{2}{*}{21} & \multirow[t]{2}{*}{ Maranhão } & 7.67 & 9.22 & 20.33 & 13.30 & 21.69 & 63.02 & 221.06 & 273.68 & 23.81 \\
\hline & & $6.51 ; 9.02$ & $\begin{array}{l}8.41 \\
10.03\end{array}$ & $0.90 ; 43.88$ & $\begin{array}{l}11.04 ; \\
15.77\end{array}$ & $\begin{array}{l}19.49 ; \\
23.78\end{array}$ & $\begin{array}{l}33.53 \\
98.71\end{array}$ & $\begin{array}{l}185.99 \\
260.07\end{array}$ & $\begin{array}{l}249.38 \\
298.48\end{array}$ & $2.62 ; 50.43$ \\
\hline \multirow[t]{2}{*}{22} & \multirow[t]{2}{*}{ Piauí } & 9.08 & 10.26 & 12.97 & 18.23 & 25.93 & 42.27 & 277.06 & 313.61 & 13.19 \\
\hline & & $8.11 ; 10.20$ & $\begin{array}{l}9.43 \\
11.13\end{array}$ & $\begin{array}{l}-2.51 \\
30.83\end{array}$ & $\begin{array}{l}16.07 \\
20.69\end{array}$ & $\begin{array}{l}23.49 ; \\
28.49\end{array}$ & $\begin{array}{l}20.94 ; \\
67.69\end{array}$ & $\begin{array}{l}245.43 \\
313.69\end{array}$ & $\begin{array}{l}286.57 ; \\
340.58\end{array}$ & $\begin{array}{l}-2.83 ; \\
32.68\end{array}$ \\
\hline \multirow[t]{2}{*}{23} & \multirow[t]{2}{*}{ Ceará } & 14.57 & 13.30 & -8.74 & 28.83 & 34.25 & 18.81 & 443.67 & 396.94 & -10.53 \\
\hline & & $13.04 ; 16.14$ & $\begin{array}{l}12.44 ; \\
14.20\end{array}$ & $-19.94 ; 3.44$ & $\begin{array}{l}25.53 ; \\
32.29\end{array}$ & $\begin{array}{l}31.33 ; \\
37.36\end{array}$ & $2.32 ; 38.40$ & $\begin{array}{l}395.69 ; \\
495.24\end{array}$ & $\begin{array}{l}369.18 \\
427.86\end{array}$ & $\begin{array}{l}-21.76 \\
1.88\end{array}$ \\
\hline 24 & Rio Grande do & 10.39 & 13.26 & 27.56 & 19.99 & 36.38 & 82.01 & 304.96 & 395.85 & 29.80 \\
\hline & & $9.39 ; 11.41$ & $\begin{array}{l}12.30 \\
14.27\end{array}$ & $13.04 ; 44.0$ & $\begin{array}{l}17.80 \\
22.28\end{array}$ & $\begin{array}{l}33.02 ; \\
39.74\end{array}$ & $\begin{array}{l}58.55 \\
109.89\end{array}$ & $\begin{array}{l}272.42 \\
337.04\end{array}$ & $\begin{array}{l}364.67 ; \\
431.10\end{array}$ & $13.76 ; 47.56$ \\
\hline 25 & Paraíba & 12.83 & 12.71 & -0.93 & 24.40 & 33.00 & 35.22 & 379.53 & 375.08 & -1.17 \\
\hline & & $11.62 ; 14.17$ & $\begin{array}{l}11.35 ; \\
14.22\end{array}$ & $\begin{array}{l}-14.72 \\
16.15\end{array}$ & $\begin{array}{l}21.74 ; \\
27.07\end{array}$ & $\begin{array}{l}29.04 ; \\
37.18\end{array}$ & $\begin{array}{l}13.86 \\
60.02\end{array}$ & $\begin{array}{l}340.87 \\
420.58\end{array}$ & $\begin{array}{l}330.96 ; \\
423.68\end{array}$ & $\begin{array}{l}-15.19 ; \\
16.98\end{array}$ \\
\hline 26 & Pernambuco & 14.84 & 15.31 & 3.13 & 25.93 & 37.79 & 45.71 & 432.17 & 448.36 & 3.75 \\
\hline & & $14.02 ; 15.72$ & $\begin{array}{l}14.37 \\
16.30\end{array}$ & $\begin{array}{l}-5.30 \\
12.09\end{array}$ & $\begin{array}{l}24.16 ; \\
27.76\end{array}$ & $\begin{array}{l}34.69 ; \\
41.01\end{array}$ & $\begin{array}{l}30.40 \\
62.23\end{array}$ & $\begin{array}{l}404.79 \\
460.62\end{array}$ & $\begin{array}{l}418.13 \\
480.12\end{array}$ & $\begin{array}{l}-5.04 \\
13.82\end{array}$ \\
\hline 27 & Alagoas & 8.99 & 11.60 & 28.97 & 15.04 & 28.20 & 87.52 & 266.13 & 347.90 & 30.73 \\
\hline & & $8.26 ; 9.88$ & 10.68 & $12.37 ; 47.11$ & 13.63; & 25.74 & 61.97; & 242.16 & $318.95 ;$ & $14.14 ; 50.31$ \\
\hline
\end{tabular}


Table 1 Age-standardized female breast cancer death (ASDR), incidence (ASIR) and disability-adjusted life years (DALYs) rates according to Brazilian states and regions in 1990 and 2017, and percentage change (D\%) (Continued)

\begin{tabular}{|c|c|c|c|c|c|c|c|c|c|c|}
\hline \multirow{2}{*}{$\begin{array}{l}\text { IBGE } \\
\text { CODE }\end{array}$} & \multirow[b]{2}{*}{ REGION/UF } & \multicolumn{3}{|l|}{$\begin{array}{l}\text { ASDR } \\
\text { (UI 95\%) }\end{array}$} & \multicolumn{3}{|c|}{$\begin{array}{c}\text { ASIR } \\
(\mathrm{UI} 95 \%)\end{array}$} & \multicolumn{3}{|c|}{$\begin{array}{l}\text { DALY } \\
(\mathrm{UI} 95 \%) \\
\end{array}$} \\
\hline & & 1990 & 2017 & $\Delta \%$ & 1990 & 2017 & $\Delta \%$ & 1990 & 2017 & $\Delta \%$ \\
\hline & & & 12.58 & & 16.68 & 30.96 & 116.65 & 295.02 & 379.90 & \\
\hline \multirow[t]{2}{*}{28} & Sergipe & 11.53 & 13.56 & 17.63 & 20.63 & 34.74 & 68.40 & 331.69 & 404.14 & 21.84 \\
\hline & & $10.53 ; 12.48$ & $\begin{array}{l}12.50 \\
14.65\end{array}$ & $4.73 ; 30.85$ & $\begin{array}{l}18.80 ; \\
22.52\end{array}$ & $\begin{array}{l}31.42 \\
37.97\end{array}$ & $\begin{array}{l}48.06 ; \\
90.93\end{array}$ & $\begin{array}{l}301.80 \\
361.61\end{array}$ & $\begin{array}{l}370.64 \\
438.10\end{array}$ & $7.35 ; 37.39$ \\
\hline \multirow[t]{2}{*}{29} & Bahia & 10.39 & 12.12 & 16.69 & 19.00 & 31.75 & 67.06 & 311.06 & 378.90 & 21.81 \\
\hline & & $9.47 ; 11.30$ & $\begin{array}{l}11.43 \\
12.96\end{array}$ & $5.19 ; 30.25$ & $\begin{array}{l}17.22 \\
20.77\end{array}$ & $\begin{array}{l}29.24 \\
34.63\end{array}$ & $\begin{array}{l}47.30 \\
89.98\end{array}$ & $\begin{array}{l}283.17 \\
338.92\end{array}$ & $\begin{array}{l}353.84 \\
407.68\end{array}$ & $9.09 ; 37.24$ \\
\hline 3 & Southeast region & & & & & & & & & \\
\hline \multirow[t]{2}{*}{31} & Minas Gerais & 12.75 & 13.18 & 3.42 & 23.71 & 38.03 & 60.41 & 364.47 & 398.10 & 9.23 \\
\hline & & $12.14 ; 13.33$ & $\begin{array}{l}12.40 \\
13.94\end{array}$ & $\begin{array}{l}-3.48 \\
10.85\end{array}$ & $\begin{array}{l}22.25 \\
25.12\end{array}$ & $\begin{array}{l}35.11 \\
41.10\end{array}$ & $\begin{array}{l}45.29 \\
76.40\end{array}$ & $\begin{array}{l}345.73 \\
383.72\end{array}$ & $\begin{array}{l}372.63 \\
424.72\end{array}$ & $0.99 ; 17.87$ \\
\hline \multirow[t]{2}{*}{32} & Espírito Santo & 12.53 & 12.34 & -1.57 & 23.77 & 34.96 & 47.06 & 357.71 & 368.92 & 3.14 \\
\hline & & $11.84 ; 13.27$ & $\begin{array}{l}11.43 ; \\
13.22\end{array}$ & $-10.25 ; 7.26$ & $\begin{array}{l}22.16 \\
25.50\end{array}$ & $\begin{array}{l}31.70 \\
38.23\end{array}$ & $\begin{array}{l}31.21 \\
63.84\end{array}$ & $\begin{array}{l}336.58 \\
381.76\end{array}$ & $\begin{array}{l}340.48 \\
399.47\end{array}$ & $\begin{array}{l}-6.95 \\
12.92\end{array}$ \\
\hline \multirow[t]{2}{*}{33} & Rio de Janeiro & 21.50 & 19.84 & -7.73 & 41.54 & 56.72 & 36.53 & 608.30 & 579.95 & -4.66 \\
\hline & & $20.65 ; 22.40$ & $\begin{array}{l}18.65 \\
21.06\end{array}$ & $\begin{array}{l}-14.01 \\
-0.69\end{array}$ & $\begin{array}{l}39.30 ; \\
44.13\end{array}$ & $\begin{array}{l}52.37 ; \\
61.55\end{array}$ & $\begin{array}{l}24.09 \\
50.45\end{array}$ & $\begin{array}{l}581.98 \\
636.69\end{array}$ & $\begin{array}{l}542.42 \\
617.83\end{array}$ & $\begin{array}{l}-11.54 \\
2.94\end{array}$ \\
\hline \multirow[t]{2}{*}{35} & São Paulo & 18.73 & 16.06 & -14.26 & 37.88 & 49.96 & 31.88 & 528.36 & 466.40 & -11.73 \\
\hline & & $18.03 ; 19.41$ & $\begin{array}{l}15.24 \\
16.90\end{array}$ & $\begin{array}{l}-19.84 \\
-8.73\end{array}$ & $\begin{array}{l}35.87 \\
40.10\end{array}$ & $\begin{array}{l}46.20 \\
54.24\end{array}$ & $\begin{array}{l}20.41 \\
45.69\end{array}$ & $\begin{array}{l}507.34 \\
551.97\end{array}$ & $\begin{array}{l}439.47 \\
496.48\end{array}$ & $\begin{array}{l}-17.90 \\
-5.42\end{array}$ \\
\hline 4 & South region & & & & & & & & & \\
\hline \multirow[t]{2}{*}{41} & Paraná & 14.50 & 15.41 & 6.28 & 27.28 & 43.94 & 61.04 & 403.36 & 447.12 & 10.85 \\
\hline & & $13.85 ; 15.21$ & $\begin{array}{l}14.52 \\
16.36\end{array}$ & $\begin{array}{l}-1.25 \\
14.29\end{array}$ & $\begin{array}{l}25.65 \\
28.98\end{array}$ & $\begin{array}{l}40.40 ; \\
47.81\end{array}$ & $\begin{array}{l}46.76 ; \\
78.62\end{array}$ & $\begin{array}{l}383.92 ; \\
424.23\end{array}$ & $\begin{array}{l}417.06 ; \\
478.25\end{array}$ & $2.03 ; 19.81$ \\
\hline \multirow[t]{2}{*}{42} & Santa Catarina & 14.59 & 15.24 & 4.45 & 28.79 & 47.27 & 64.16 & 404.16 & 448.67 & 11.01 \\
\hline & & $13.91 ; 15.33$ & $\begin{array}{l}14.31 \\
16.31\end{array}$ & $-4.40 ; 13.38$ & $\begin{array}{l}26.99 \\
30.68\end{array}$ & $\begin{array}{l}43.45 \\
51.57\end{array}$ & $\begin{array}{l}48.05 \\
83.55\end{array}$ & $\begin{array}{l}382.13 \\
425.23\end{array}$ & $\begin{array}{l}417.09 ; \\
482.49\end{array}$ & $1.31 ; 21.39$ \\
\hline \multirow[t]{2}{*}{43} & Rio Grande do Sul & 20.41 & 17.54 & -14.08 & 41.62 & 52.77 & 26.80 & 573.63 & 503.47 & -12.23 \\
\hline & & $19.62 ; 21.24$ & $\begin{array}{l}16.50 \\
18.62\end{array}$ & $\begin{array}{l}-20.22 \\
-7.54\end{array}$ & $\begin{array}{l}39.23 \\
44.09\end{array}$ & $\begin{array}{l}48.53 \\
57.58\end{array}$ & $\begin{array}{l}14.58 \\
40.22\end{array}$ & $\begin{array}{l}548.26 \\
599.47\end{array}$ & $\begin{array}{l}469.51 \\
538.79\end{array}$ & $\begin{array}{l}-18.94 \\
-5.03\end{array}$ \\
\hline 5 & $\begin{array}{l}\text { Central-West } \\
\text { region }\end{array}$ & & & & & & & & & \\
\hline \multirow[t]{2}{*}{50} & Mato Grosso do & 12.70 & 13.60 & 7.09 & 23.93 & 36.79 & 53.77 & 366.29 & 398.71 & 8.85 \\
\hline & sul & $11.87 ; 13.57$ & $\begin{array}{l}12.67 ; \\
14.71\end{array}$ & $\begin{array}{l}-2.63 ; \\
18.38\end{array}$ & $\begin{array}{l}22.14 ; \\
25.91\end{array}$ & $\begin{array}{l}33.63 ; \\
40.43\end{array}$ & $\begin{array}{l}36.71 ; \\
73.27\end{array}$ & $\begin{array}{l}340.52 ; \\
393.30\end{array}$ & $\begin{array}{l}369.65 \\
431.17\end{array}$ & $\begin{array}{l}-1.95 \\
21.26\end{array}$ \\
\hline \multirow[t]{2}{*}{51} & Mato Grosso & 9.50 & 11.03 & 16.07 & 18.11 & 29.79 & 64.48 & 284.19 & 323.36 & 13.79 \\
\hline & & $8.50 ; 10.55$ & $\begin{array}{l}10.10 \\
11.99\end{array}$ & $1.43 ; 34.21$ & $\begin{array}{l}16.10 \\
20.20\end{array}$ & $\begin{array}{l}26.96 \\
33.00\end{array}$ & $\begin{array}{l}40.79 ; \\
92.65\end{array}$ & $\begin{array}{l}252.49 \\
316.43\end{array}$ & $\begin{array}{l}295.46 \\
352.88\end{array}$ & $\begin{array}{l}-1.00 \\
32.40\end{array}$ \\
\hline \multirow[t]{2}{*}{52} & Goiás & 13.13 & 11.98 & -8.75 & 23.91 & 32.96 & 37.83 & 366.05 & 357.80 & -2.25 \\
\hline & & $12.37 ; 13.88$ & $\begin{array}{l}11.14 \\
12.86\end{array}$ & $\begin{array}{l}-16.39 \\
-0.45\end{array}$ & $\begin{array}{l}22.19 ; \\
25.62\end{array}$ & $\begin{array}{l}30.25 \\
36.09\end{array}$ & $\begin{array}{l}24.20 \\
54.08\end{array}$ & $\begin{array}{l}344.56 \\
387.99\end{array}$ & $\begin{array}{l}332.50 \\
386.87\end{array}$ & $\begin{array}{l}-11.26 \\
6.52\end{array}$ \\
\hline \multirow[t]{2}{*}{53} & Distrito Federal & 17.34 & 14.79 & -14.71 & 36.71 & 49.83 & 35.76 & 478.06 & 393.29 & -17.73 \\
\hline & & $16.32 ; 18.40$ & $\begin{array}{l}13.62 \\
16.02\end{array}$ & $\begin{array}{l}-22.66 \\
-5.40\end{array}$ & $\begin{array}{l}34.17 \\
39.26\end{array}$ & $\begin{array}{l}45.16 \\
55.15\end{array}$ & $\begin{array}{l}20.83 \\
53.88\end{array}$ & $\begin{array}{l}446.91 ; \\
508.18\end{array}$ & $\begin{array}{l}361.20 \\
426.18\end{array}$ & $\begin{array}{l}-25.29 \\
-8.90\end{array}$ \\
\hline
\end{tabular}




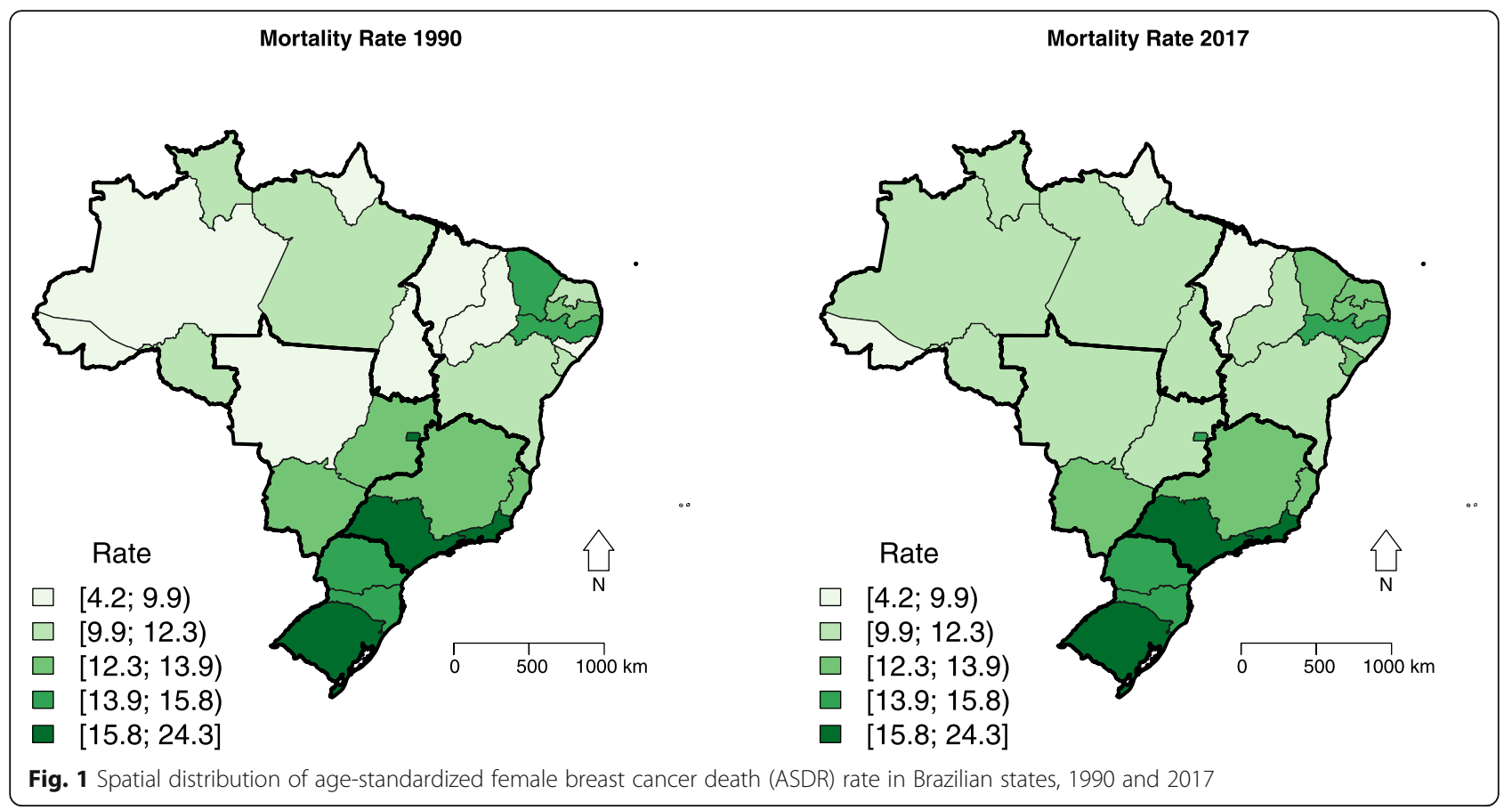

Clusters of states with low SDI values were identified in the Northeast region and clusters of states with high SDI values were found in the Southeast and South regions. The clusters for mortality, incidence, and DALYs rates showed similar patterns, with low values in the North and Northeast regions and high values in the South and Southeast regions. On the other hand, when considering the percentage change of all these indicators, clusters of high values were observed in the North and Northeast regions, and of low values in the South and Southeast regions. However, MIR had different behavior, with clusters of low values in the South and Southeast regions, and high values in the North and Northeast regions, where there were also the lowest reductions in

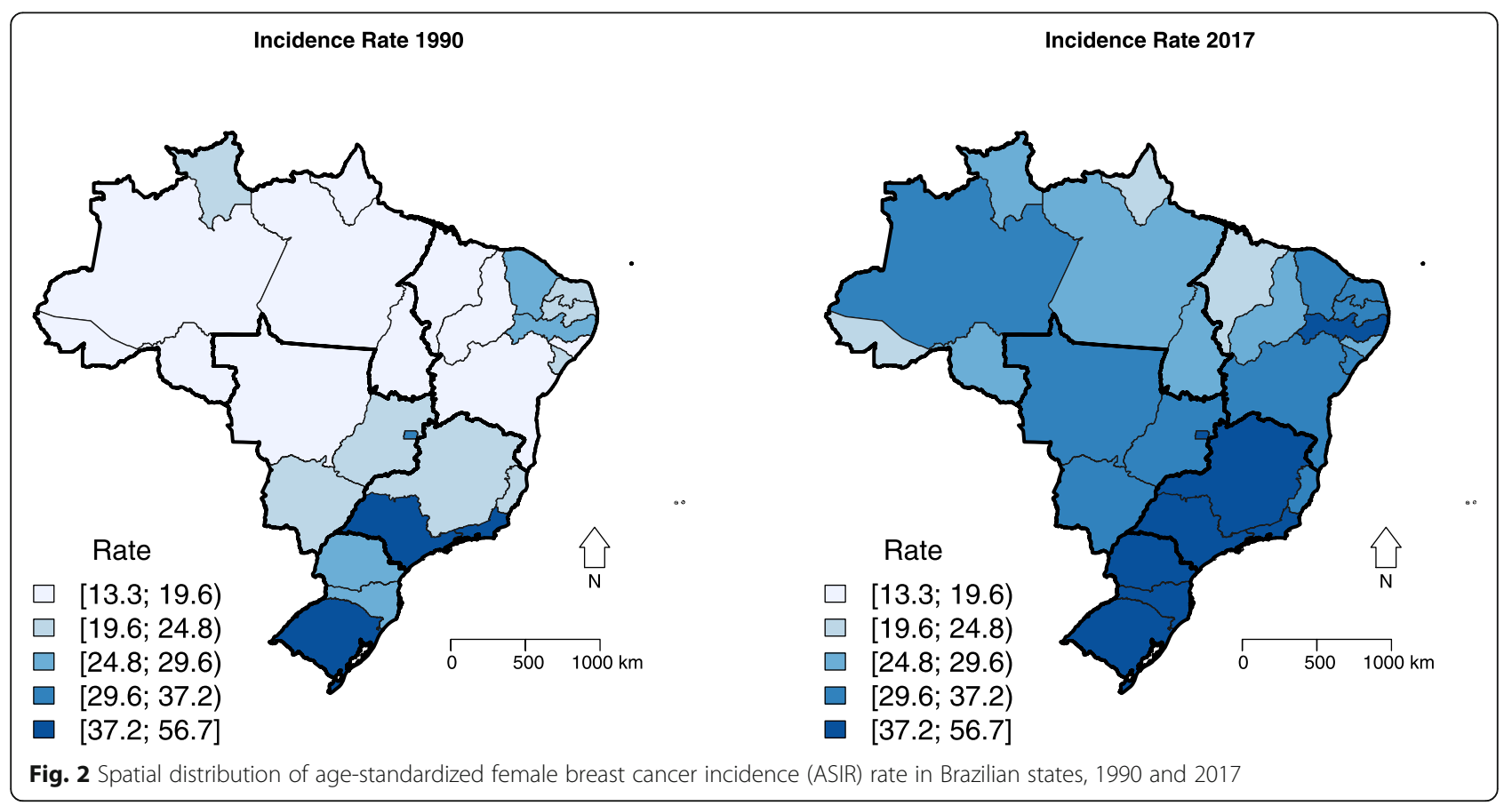


Table 2 Socio-demographic index (SDI) and female breast cancer mortality-to-incidence (MIR) ratio according to Brazilian states and regions in 1990 and 2017, and the percentage change $(\Delta \%)$

\begin{tabular}{|c|c|c|c|c|c|c|c|}
\hline & & SDI & & & MIR & & \\
\hline IBGE CODE & REGION/UF & 1990 & 2017 & $\Delta \%$ & 1990 & 2017 & $\Delta \%$ \\
\hline - & Brazil & 0.49 & 0.66 & 34.30 & 0.51 & 0.35 & -31.75 \\
\hline 1 & North region & & & & & & \\
\hline 11 & Rondônia & 0.42 & 0.62 & 46.82 & 0.56 & 0.37 & -33.59 \\
\hline 12 & Acre & 0.38 & 0.60 & 58.74 & 0.56 & 0.42 & -25.69 \\
\hline 13 & Amazonas & 0.44 & 0.63 & 42.81 & 0.54 & 0.38 & -29.94 \\
\hline 14 & Roraima & 0.43 & 0.65 & 50.09 & 0.57 & 0.41 & -28.14 \\
\hline 15 & Pará & 0.41 & 0.58 & 40.17 & 0.54 & 0.40 & -25.36 \\
\hline 16 & Amapá & 0.47 & 0.66 & 40.19 & 0.51 & 0.39 & -24.19 \\
\hline 17 & Tocantins & 0.40 & 0.61 & 53.29 & 0.52 & 0.38 & -27.83 \\
\hline 2 & Northeast region & & & & & & \\
\hline 21 & Maranhão & 0.31 & 0.51 & 60.71 & 0.58 & 0.43 & -26.18 \\
\hline 22 & Piauí & 0.37 & 0.55 & 49.93 & 0.50 & 0.40 & -20.59 \\
\hline 23 & Ceará & 0.41 & 0.60 & 44.89 & 0.51 & 0.39 & -23.19 \\
\hline 24 & Rio Grande do Norte & 0.42 & 0.61 & 44.92 & 0.52 & 0.36 & -29.91 \\
\hline 25 & Paraíba & 0.40 & 0.57 & 43.06 & 0.53 & 0.39 & -26.73 \\
\hline 26 & Pernambuco & 0.42 & 0.59 & 41.68 & 0.57 & 0.41 & -29.22 \\
\hline 27 & Alagoas & 0.36 & 0.56 & 55.26 & 0.60 & 0.41 & -31.22 \\
\hline 28 & Sergipe & 0.42 & 0.62 & 44.01 & 0.56 & 0.39 & -30.15 \\
\hline 29 & Bahia & 0.40 & 0.59 & 46.20 & 0.55 & 0.38 & -30.15 \\
\hline 3 & Southeast region & & & & & & \\
\hline 31 & Minas Gerais & 0.49 & 0.66 & 33.90 & 0.54 & 0.35 & -35.53 \\
\hline 32 & Espírito Santo & 0.50 & 0.68 & 34.91 & 0.53 & 0.35 & -33.07 \\
\hline 33 & Rio de Janeiro & 0.58 & 0.71 & 22.53 & 0.52 & 0.35 & -32.42 \\
\hline 35 & São Paulo & 0.56 & 0.72 & 28.54 & 0.49 & 0.32 & -34.99 \\
\hline 4 & South region & & & & & & \\
\hline 41 & Paraná & 0.51 & 0.68 & 32.39 & 0.53 & 0.35 & -34.00 \\
\hline 42 & Santa Catarina & 0.54 & 0.70 & 29.14 & 0.51 & 0.32 & -36.37 \\
\hline 43 & Rio Grande do Sul & 0.54 & 0.69 & 26.94 & 0.49 & 0.33 & -32.24 \\
\hline 5 & Central-West region & & & & & & \\
\hline 50 & Mato Grosso do Sul & 0.46 & 0.65 & 39.04 & 0.53 & 0.37 & -30.36 \\
\hline 51 & Mato Grosso & 0.47 & 0.66 & 38.73 & 0.52 & 0.37 & -29.43 \\
\hline 52 & Goiás & 0.46 & 0.65 & 40.62 & 0.55 & 0.36 & -33.79 \\
\hline 53 & Distrito Federal & 0.63 & 0.79 & 25.26 & 0.47 & 0.30 & -37.18 \\
\hline
\end{tabular}

this indicator between 1990 and 2017 years, highlighting regional differences (Fig. 6).

In all the panel models, the Pesaran test was significant, indicating cross-sectional dependence and pointing the spatial models for adjusting the spatial correlation. The Hausman test was significant for models with ASDR, ASIR and DALYs as outcome variables, guiding the choice of fixed effect spatial panel models. As the model for MIR had non-significant Hausman test, the most appropriate choice was the spatial random effect model. The SDI had a positive association with incidence rate $(\operatorname{coef}=30.38, \quad p<0.001$; Table 5) and a negative association with MIR (coef = $-0.212, p<0.001$; Table 5 ), showing no association with mortality rate.

\section{Discussion}

In Brazil, breast cancer mortality rate had a modest decrease (-4.45\%) between 1990 and 2017 years, while the incidence rate has increased substantially (+39.99\%). 


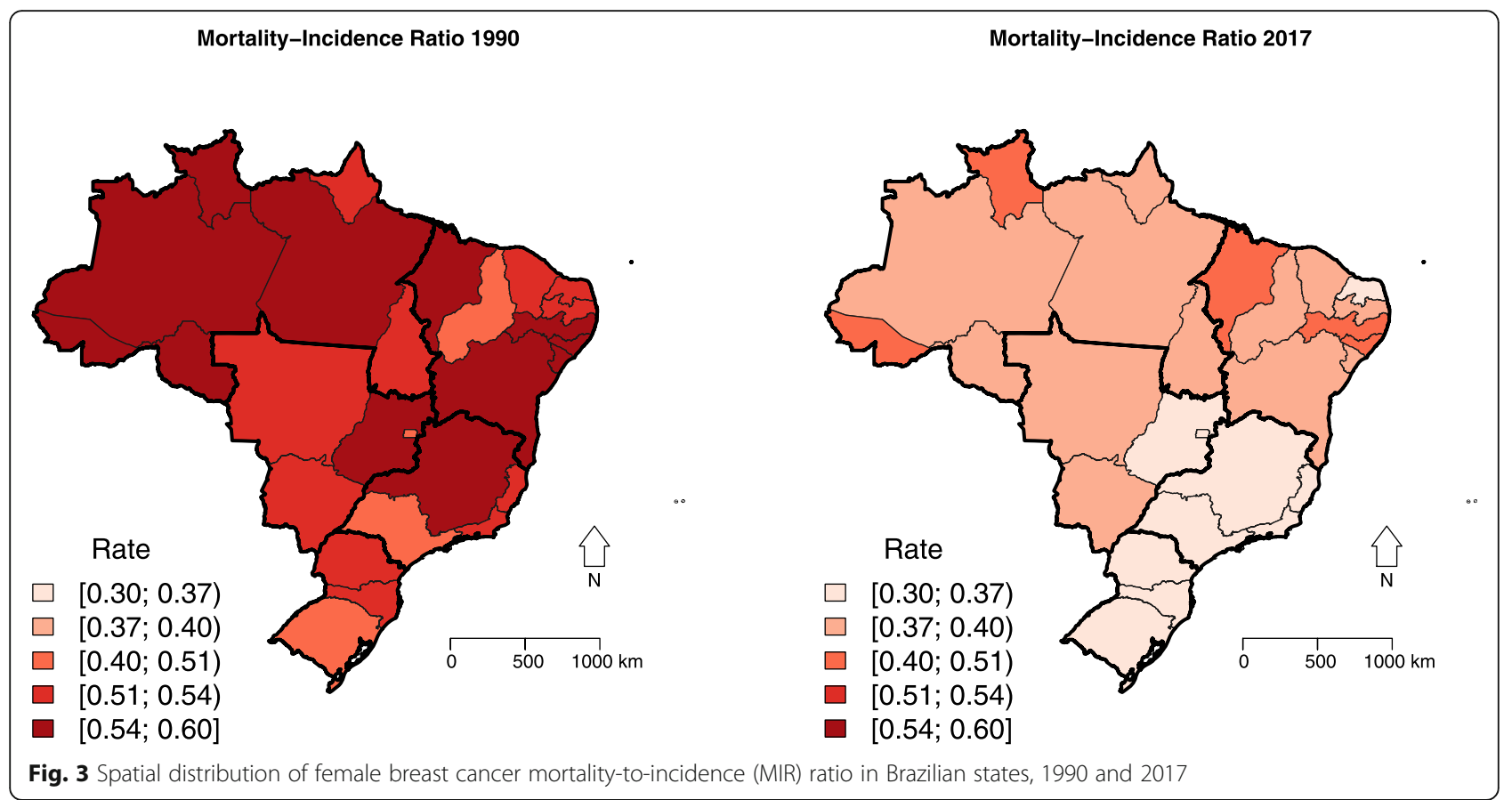

Furthermore, the MIR for breast cancer declined in the country between 1990 and 2017 years $(-31.75 \%)$. The MIR, a proxy for survival rat e[5, 22], provides an alternative means to assess the burden of a disease. It is useful to identify inequities in cancer outcomes, enabling evaluation of cancer control programs, including cancer screening and treatment [29]. The SDI showed an increase in all Brazilian states in the same period, notably in less developed regions, showing a positive association with incidence rate and a negative association with MIR. Such findings reinforce the influence of sociodemographic improvement on the increase of breast cancer incidence. It also suggests an improvement in breast cancer survival in the period, which may be

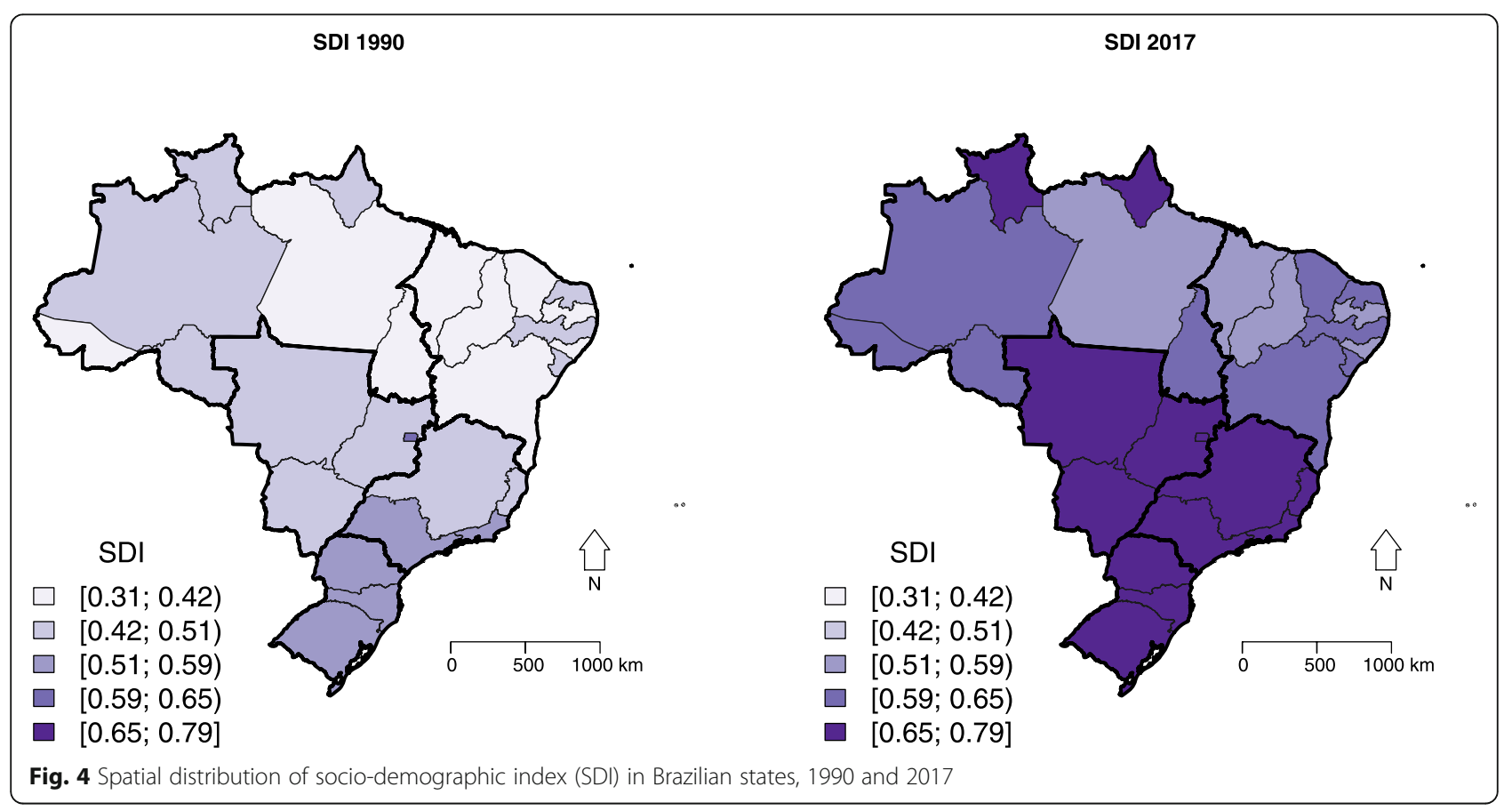




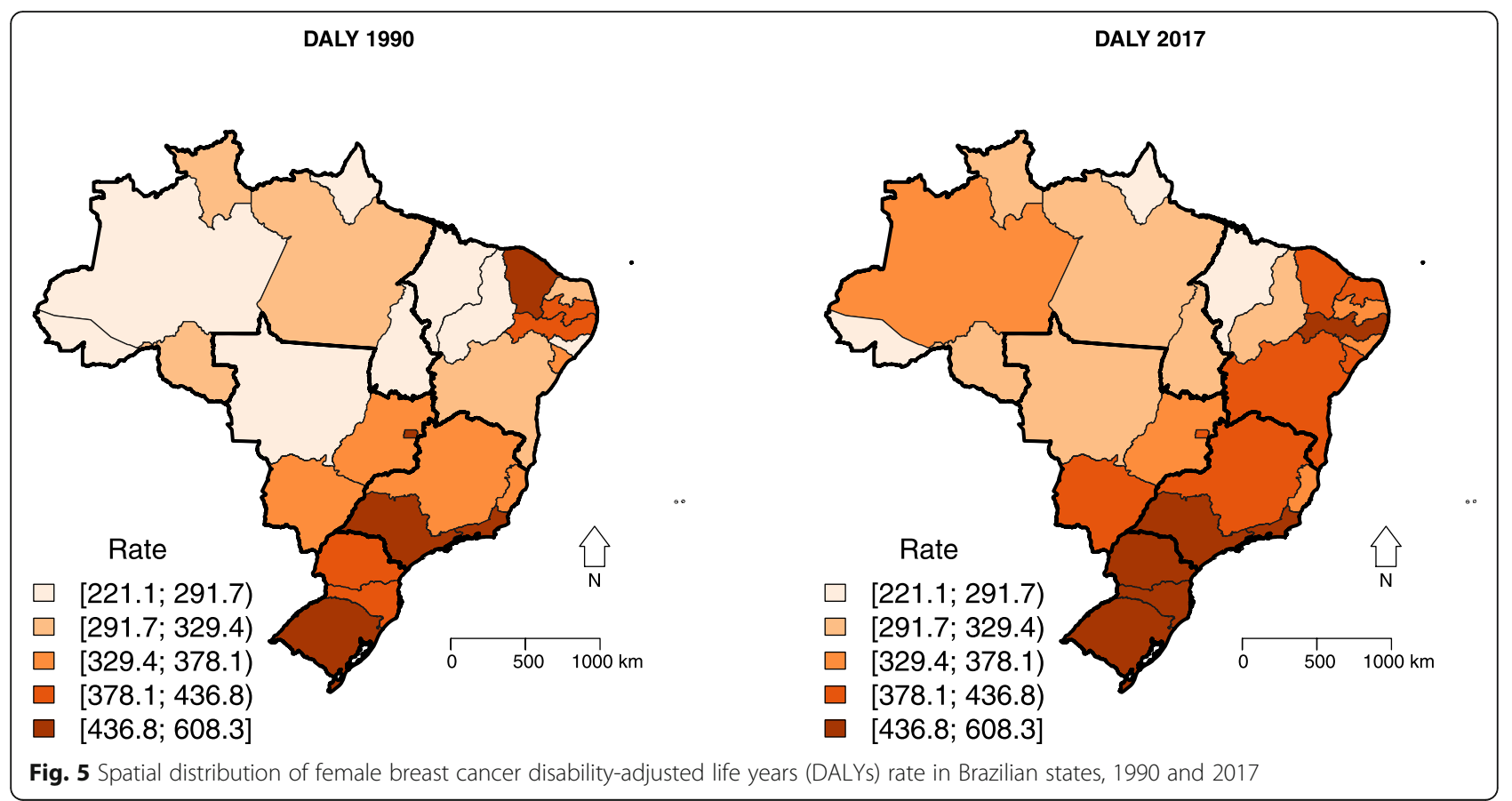

related to a broader access to diagnostic methods and recommended treatment. It is noteworthy, however, that the regions with the worst socioeconomic conditions showed a lower reduction of MIR, which is an indirect estimation of survival $[5,22]$. The long-term survival of women with breast cancer has been increasing in Brazil, although it still shows lower rates than in higher-income countries. This increase in survival has been attributed to improvements in breast cancer treatment, and also to the increase in mammographic screening [30]. However, a study that analyzed data from hospital-based cancer registries in Brazil did not show a reduction in the late

Table 3 Pearson correlation coefficient between sociodemographic index (SDI), age-standardized female breast cancer death (ASDR), incidence (ASIR) and disability-adjusted life years (DALYs) rates and female breast cancer mortality-to-incidence (MIR) ratio, Brazil, 1990 and 2017

\begin{tabular}{lllllll}
\hline Year & Variables & SDI & ASDR & ASIR & DALY & MIR \\
\hline 1990 & SDI & 1 & 0.79 & 0.82 & 0.76 & -0.68 \\
& ASDR & 0.79 & 1 & 0.99 & 1.00 & -0.52 \\
& ASIR & 0.82 & 0.99 & 1 & 0.99 & -0.63 \\
& DALY & 0.76 & 1.00 & 0.99 & 1 & -0.52 \\
& MIR & -0.68 & -0.52 & -0.63 & -0.52 & 1 \\
& SDI & 1 & 0.61 & 0.76 & 0.53 & -0.87 \\
& ASDR & 0.61 & 1 & 0.96 & 0.99 & -0.65 \\
& ASIR & 0.76 & 0.96 & 1 & 0.93 & -0.82 \\
& DALY & 0.53 & 0.99 & 0.93 & 1 & -0.61 \\
& MIR & -0.87 & -0.65 & -0.82 & -0.61 & 1 \\
\hline
\end{tabular}

All $p$ values $<0.05$ stage of breast cancer, even with the implementation of the screening national program in 2004 [31].

Brazilian studies of the trends in breast cancer mortality in the last decades show stability $[8,9]$ or even increase [10-14] in deaths due to breast cancer in Brazil. The variation in the findings is likely a function of the type of records included and the years considered. However, most studies used data from the Ministry of Health Information Systems (DATASUS) with the most recent follow-up, at most, until the year 2014. Even studies that found a trend of increased mortality are in accordance with the present findings with regard to the regional disparity of breast

Table 4 Spatial autocorrelation coefficients (Moran's I) for sociodemographic index (SDI), age-standardized female breast cancer death (ASDR), incidence (ASIR) and disability-adjusted life years (DALYs) rates and female breast cancer mortality-to-incidence (MIR) ratio, Brazilian states, 1990 and 2017

\begin{tabular}{llll}
\hline Variables & Year & Moran's I & $p$ value \\
\hline SDI & 1990 & 0.581 & 0.001 \\
& 2017 & 0.579 & 0.001 \\
ASDR & 1990 & 0.424 & 0.001 \\
& 2017 & 0.496 & 0.001 \\
ASIR & 1990 & 0.436 & 0.001 \\
& 2017 & 0.581 & 0.001 \\
DALY & 1990 & 0.387 & 0.001 \\
& 2017 & 0.525 & 0.001 \\
MIR & 1990 & 0.156 & 0.052 \\
& 2017 & 0.577 & 0.001 \\
\hline${ }^{*} p$ value obtained per 1000 Monte-Carlo simulations; significant if $p<0.05$
\end{tabular}




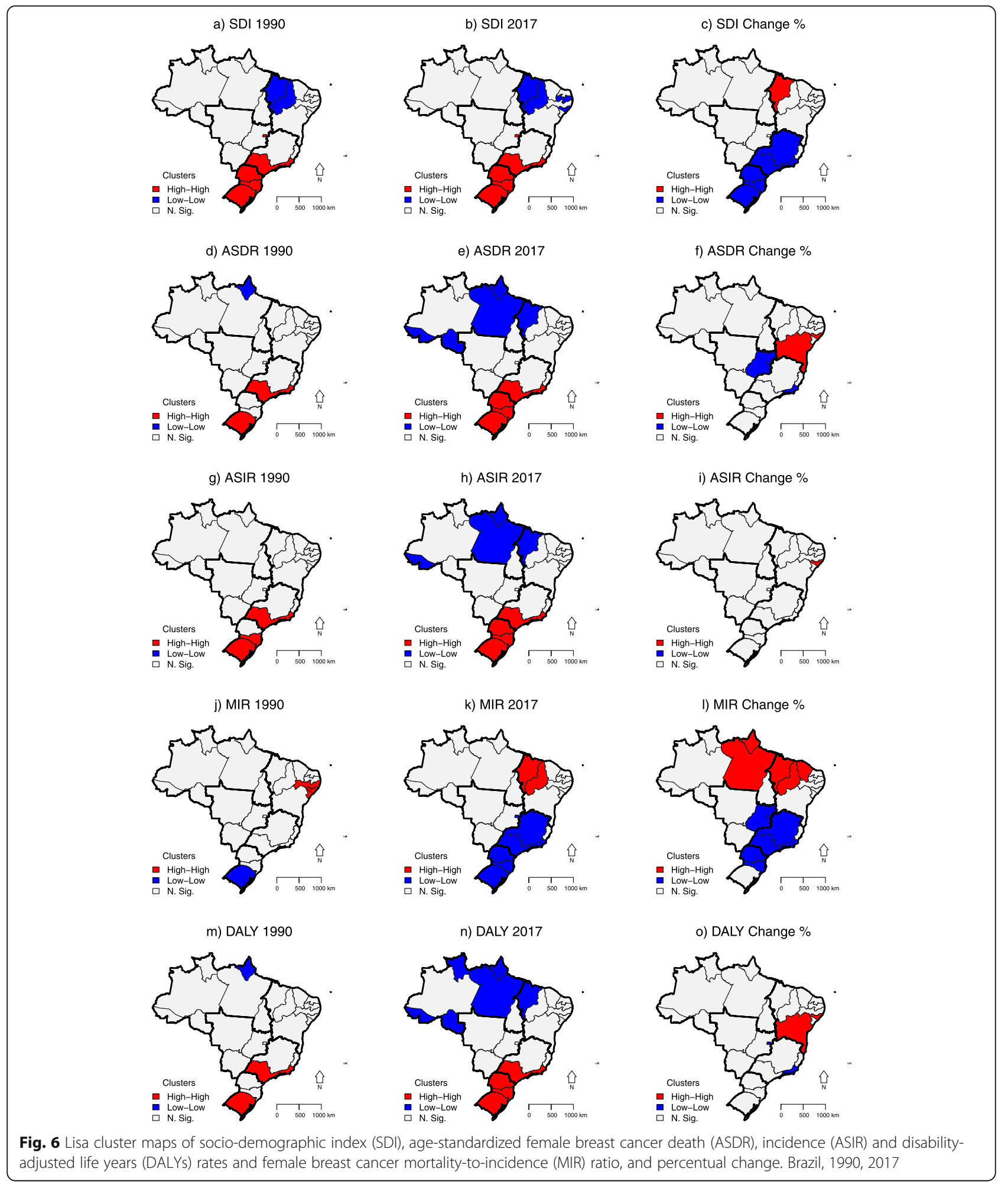

cancer mortality in Brazil. A study carried out with the mortality information system data from 1980 to 2009 found stability of breast cancer mortality in Brazil since 1994, with a tendency to increase in the North, Northeast, and Central West regions, stability in the South region and reduction in the Southeast region. The risk of death from breast cancer was at least twice as high in states such as São Paulo, Rio de Janeiro, Rio Grande do Sul, and the Federal District, which have higher Human Development Index (HDI), in comparison to the States of Alagoas, 
Table 5 Panel models results for the association between sociodemographic index (SDI) and age-standardized female breast cancer death (ASDR), incidence (ASIR) and disability-adjusted life years (DALYS) rates and female breast cancer mortality-toincidence (MIR) ratio in Brazilian states, 1990-2017

\begin{tabular}{|c|c|c|c|}
\hline Panel models & Coef. SDI & Std. error & $p$ value \\
\hline \multicolumn{4}{|l|}{$\mathrm{ASDR}^{\mathrm{a}}$} \\
\hline Pooled model & 20.390 & 1.194 & $<0.001$ \\
\hline Fixed effect model & -0.102 & 0.032 & 0.866 \\
\hline Random effect model & 0.166 & 0.610 & 0.786 \\
\hline Spatial fixed effect lag model & 0.079 & 0.560 & 0.888 \\
\hline Spatial random effect lag model & 0.226 & 0.568 & 0.690 \\
\hline \multicolumn{4}{|l|}{$A S I R^{b}$} \\
\hline Pooled model & 86.500 & 2.685 & $<0.001$ \\
\hline Fixed effect model & 55.815 & 1.363 & $<0.001$ \\
\hline Random effect model & 56.147 & 1.365 & $<0.001$ \\
\hline Spatial fixed effect lag model & 30.382 & 2.423 & $<0.001$ \\
\hline Spatial random effect lag model & 30.707 & 1.221 & $<0.001$ \\
\hline \multicolumn{4}{|l|}{ DALYc } \\
\hline Pooled model & 520.538 & 33.847 & $<0.001$ \\
\hline Fixed effect model & 26.597 & 17.385 & 0.127 \\
\hline Random effect model & 32.640 & 17.498 & 0.063 \\
\hline Spatial fixed effect lag model & 21.910 & 16.328 & 0.180 \\
\hline Spatial random effect lag model & 25.628 & 16.496 & 0.120 \\
\hline \multicolumn{4}{|l|}{$M I R^{d}$} \\
\hline Pooled model & -0.539 & 0.012 & $<0.001$ \\
\hline Fixed effect model & -0.834 & 0.009 & $<0.001$ \\
\hline Random effect model & -0.814 & 0.010 & $<0.001$ \\
\hline Spatial fixed effect lag model & -0.207 & 0.018 & $<0.001$ \\
\hline Spatial random effect lag model & -0.212 & 0.006 & $<0.001$ \\
\hline
\end{tabular}

a Panel models for ASDR. Hausman test: chisq $=8.23 ; p=0.004$. Pesaran test: $z=15.23 ; p<0.001$. Selected model: spatial fixed effect lag model ${ }^{\mathrm{b}}$ Panel models for ASIR. Hausman test: $\mathrm{chisq}=13.99 ; p<0.001$. Pesaran test: $z=21.74 ; p<0.001$. Selected model: spatial fixed effect lag model 'Panel models for DALYs. Hausman test: chisq $=43.63 ; p<0.0021$. Pesaran test: $z=53.74 ; p<0.001$. Selected model: spatial fixed effect lag model

dPanel models for MIR. Hausman test: chisq $=2.04 ; p=0.153$. Pesaran test: $z=$ 5.07; $p<0.001$. Selected model: spatial random effect lag model

Maranhão, and Piauí [9]. In the present study, when considering the Brazilian regions and states, there was an increase in breast cancer mortality in the seven states, the less developed regions, while the greatest reductions were observed in the more developed regions. These findings were also observed in other Brazilian studies that worked on a subnational scale [8-13, 16, 32].

The increase in the breast cancer incidence in all Brazilian states between 1990 and 2017 years, with an even greater increase in the low-income states, point to the effect of the demographic and epidemiological change in Central and South America, with consequent change in disease determinants as reproductive and hormonal factors, and lifestyle factors, such as overweight and obesity, physical inactivity and alcohol consumption, and detection and mammographic screening [6, 13], mainly in less developed regions.

A recent study found that, between 1990 and 2015 years, mortality from breast cancer attributable to physical inactivity increased in Brazil (+0.77\%; 95\% U.I.: 0.27; $1.47)$ and decreased around the world $(-2.84 \%$; $95 \%$ U.I.: $-4.35 ;-0.10)$ [7].

Although the DALYs rates for breast cancer remained stable in Brazil between 1990 and 2017 years, which may be related to the improvement in treatment and even in early detection, considering that there was an increase in breast cancer incidence in Brazil in the same period, subnational data showed an increase in DALYs rates mainly in states with lower SDI, especially those in the Northeast region. Although government strategies seem to be effective against the burden of breast cancer in Brazil [13], the rising burden of this disease in Brazilian regions with the lowest SDI (North and Northeast) leads increasingly to marked regional disparities. To meet this challenge, each country must identify its specific needs and priorities [18].

The SDI increased between 1990 and 2017 years both for Brazil as a whole and for all Brazilian states, which suggests a general improvement in the level of development in the country, which affected all regions. As SDI increases, cancers associated with lifestyle factors of more developed regions, such as breast cancer, are becoming more common [19]. On the other hand, the MIR declined between 1990 and 2017 years both for Brazil in general and for all states, which may be related to the increase in breast cancer incidence faster than mortality [5], or may also point to an implementation of an effective cancer control programs, including cancer screening [29].

An important limitation of the present study is the use of estimates of the indicators at national and mainly subnational scale, which can be influenced by variability of data sources, depending on the level of regional development, with higher quality in the more developed regions. However, the GBD 2017 study makes substantial efforts to enhance the comparability of results by applying corrections for under-registration and garbage code redistribution algorithms [19].

In addition, the indicators estimated in this study, based on data from the GBD 2017 study, were very close to those estimated in other studies that used data from the national information systems, which reinforces the validity of our results.

\section{Conclusion}

This study found that there are inequalities in breast cancer outcomes among Brazilian states and regions. 
These findings may help to guide public policy priorities in the country, as well as enable an evaluation of the breast cancer control program.

The study included a follow-up of the burden of breast cancer over an extended period of time (from 1990 to 2017), which allowed a more valid prediction about breast cancer trends in Brazil.

Periodic evaluations using consistent indicators can help evaluate the success of breast cancer prevention, diagnosis, and treatment in Brazil.

\section{Supplementary information}

Supplementary information accompanies this paper at https://doi.org/10. 1186/s12963-020-00212-5.

Additional file 1: Figure S1. Brazilian Regions and States.

Additional file 2: Figure S2. Temporal distribution of age-standardized female breast cancer death (ASDR) and incidence (ASIR) rates in Brazil and Brazilian States, 1990-2017.

Additional file 3: Figure S3. Temporal distribution of socio-demographic index (SDI) and female breast cancer mortality-to-incidence (MIR) ratio in Brazil and Brazilian States, 1990-2017.

Additional file 4: Figure S4. Temporal distribution of female breast cancer disability-adjusted life years (DALYs) rate, 1990-2017.

\section{Abbreviations}

ASDR: Age-standardized breast cancer death; ASIR: Age-standardized breast cancer incidence; DALYs: Disability-adjusted life years; DATASUS: Brazilian Ministry of Health Information Systems; IBGE: Brazilian Institute of Geography and Statistics; IHME: Institute for Health Metrics and Evaluation; GBD: Global burden of disease; HDI: Human Development Index; MIR: Mortality-toincidence ratio; SDI: Socio-demographic index; UI: Uncertainty interval

\section{Acknowledgements}

The authors also acknowledge the contributions of the Brazilian Ministry of Health and the Brazilian Institute of Geography and Statistics (IBGE) staff with data exchange with the Institute of Health Metrics and Evaluation (IHME).

\section{About this supplement}

This article has been published as part of Population Health Metrics, Volume 18 Supplement 1 2020: The GBD Brazil Network. The full contents of the supplement are available at https://pophealthmetrics.biomedcentral.com/ articles/supplements/volume-18-supplement-1.

\section{Authors' contributions}

MRG and MTBT conceived the study, and MRG had a major role in the coordination of the study. MRG, MTBT, and MCN managed the data collection and performed the statistical analysis. MRG, MTBT, MCN, DCM, CSLC, MFMS, MPC, MSFM, MM, and MN participated in data interpretation and drafted the manuscript. All authors read and approved the final manuscript.

\section{Funding}

This work was supported by the Brazilian Ministry of Health through resource transfer from the National Health Fund (TED - 125/ 2017).

Publication costs are funded by the same resource: TED - 125/2017, National Health Fund/Brazilian Ministry of Health.

The funder had no role on the study design, data collection and analysis, data interpretation, and in the decision to publish.

DCM and MTBT are research fellow (Bolsista de Produtividade em Pesquisa$\mathrm{PQ}$ ) from the Conselho Nacional de Desenvolvimento Científico e

Tecnológico (CNPq/Brazil).

\section{Availability of data and materials}

The datasets supporting the conclusions of this article are publicly available online in the official website of the Institute of Health Metrics and Evaluation: http://ghdx.healthdata.org/gbd-results-tool.

Ethics approval and consent to participate

The Project "Global Burden of Diseases - GBD in Brazil" was approved by the Research Ethics Committee from the Universidade Federal de Minas Gerais (UFMG), under protocol number 62803316.7.0000.5149.

\section{Consent for publication}

Not applicable.

\section{Competing interests}

The authors declare that they have no competing interests.

\section{Author details}

${ }^{1}$ Graduate Program in Public Health, Universidade Federal de Juiz de Fora (UFJF), Juiz de Fora, Minas Gerais, Brazil. ${ }^{2}$ Department of Maternal and Child Nursing and Public Health, Nursing School, Universidade Federal de Minas Gerais (UFMG), Belo Horizonte, Minas Gerais, Brazil. ${ }^{3}$ Graduate Program of the Preventive Medicine Department, Medical School, Universidade Federal de Minas Gerais (UFMG), Belo Horizonte, Minas Gerais, Brazil. ${ }^{4}$ AC Camargo Cancer Center, AC Camargo Hospital, São Paulo, SP, Brazil. Institute for Health Metrics and Evaluation (IHME), University of Washington, Seattle, WA, USA.

Received: 15 May 2020 Accepted: 23 June 2020

Published: 30 September 2020

\section{References}

1. Ferlay J, Colombet M, Soerjomataram I, Mathers C, Parkin DM, Piñeros M, Znaor A, Bray F. Estimating the global cancer incidence and mortality in 2018: GLOBOCAN sources and methods. Int J Cancer. 2019:144(8):1941-53.

2. Bray F, Ferlay J, Soerjomataram I, Siegel RL, Torre LA, Jemal A. Global cancer statistics 2018: GLOBOCAN estimates of incidence and mortality worldwide for 36 cancers in 185 countries. CA Cancer J Clin. 2018;68(6):394-424.

3. Global Burden of Disease Cancer Collaboration, Fitzmaurice C, Akinyemiju TF, Al Lami FH, Alam T, Alizadeh-Navaei R, Allen C, et al. Global, regional, and national cancer incidence, mortality, years of life lost, years lived with disability, and disability-adjusted life-years for 29 cancer groups, 1990 to 2016: a systematic analysis for the global burden of disease study. JAMA Oncol. 2018. Nov 1:4(11):1553-68.

4. Torre LA, Siegel RL, Ward EM, Jemal A. Global cancer incidence and mortality rates and trends--an update. Cancer Epidemiol Biomarkers Prev. 2016;25(1):16-27.

5. Sharma R. Breast cancer incidence, mortality and mortality-to-incidence ratio (MIR) are associated with human development, 1990-2016: evidence from global burden of disease study 2016. Breast Cancer. 2019. Jul;26(4): 428-45.

6. Di Sibio A, Abriata G, Forman D, Sierra MS, et al. Cancer Epidemiol. 2016; 44(suppl_1):S110-20

7. Silva DAS, Tremblay MS, Souza MFM, Guerra MR, Mooney M, Naghavi M, Malta DC. Mortality and years of life lost due to breast cancer attributable to physical inactivity in the Brazilian female population (1990-2015). Sci Rep. 2018;8(1):11141.

8. Gonzaga CM, Freitas-Junior R, Curado MP, Sousa AL, Souza-Neto JA, Souza MR. Temporal trends in female breast cancer mortality in Brazil and correlations with social inequalities: ecological time-series study. BMC Public Health. 2015:15:96.

9. Freitas-Junior R, Gonzaga CM, Freitas NM, Martins E, Dardes RD. Disparities in female breast cancer mortality rates in Brazil between 1980 and 2009. Clinics (Sao Paulo). 2012;67(7):731-7.

10. Girianelli VR, Gamarra CJ, Azevedo e Silva G. Disparities in cervical and breast cancer mortality in Brazil. Rev Saude Publica. 2014:48(3):459-67.

11. Kluthcovsky AC, Faria TN, Carneiro FH, Strona R. Female breast cancer mortality in Brazil and its regions. Rev Assoc Med Bras. 2014;60(4):387-93.

12. Couto MSA, Guerra MR, Firme VAC, Bustamante-Teixeira MT. Comportamento da mortalidade por câncer de mama nos municípios brasileiros e fatores associados. Rev Panam Salud Publica. 2017;41:e168. 
13. Figueiredo FWDS, Almeida TCDC, Cardial DT, Maciel ÉDS, Fonseca FLA, Adami F. The role of health policy in the burden of breast cancer in Brazil. BMC Womens Health. 2017;17(1):121.

14. Rocha-Brischiliari SC, Oliveira RR, Andrade L, Brischiliari A, Gravena AAF, Carvalho MDB, Pelloso SM. The rise in mortality from breast cancer in young women: trend analysis in Brazil. PLoS One. 2017;12(1):e0168950.

15. Carioli G, Malvezzi M, Rodriguez T, Bertuccio P, Negri E, La Vecchia C. Trends and predictions to 2020 in breast cancer mortality: Americas and Australasia. Breast. 2018:37:163-9.

16. Guerra MR, Bustamante-Teixeira MT, Corrêa CSL, Abreu DMX, Curado MP, Mooney M, Naghavi M, Teixeira R, França EB, Malta DC. Magnitude and variation of the burden of cancer mortality in Brazil and Federation Units, 1990 and 2015. Rev Bras Epidemiol. 2017;20(suppl_01):102-15.

17. Azevedo e Silva G, de Moura L, Curado MP, Gomes FS, Otero U, LFM R, et al. The fraction of cancer attributable to ways of life, infections, occupation, and environmental agents in Brazil in 2020. PLoS One. 2016;11(2):e0148761.

18. Lee BL, Liedke PE, Barrios CH, Simon SD, Finkelstein DM, Goss PE. Breast cancer in Brazil: present status and future goals. Lancet Oncol. 2012;13(3): e95-e102.

19. GBD 2017 Causes of Death Collaborators. Global, regional, and national agesex-specific mortality for 282 causes of death in 195 countries and territories, 1980-2017: a systematic analysis for the global burden of disease study 2017. Lancet. 2018;392(10159):1736-88.

20. Ishitani LH, Teixeira RA, Abreu DMX, Paixão LMMM, França EB. Qualidade da informação das estatísticas de mortalidade: códigos garbage declarados como causas de morte em Belo Horizonte, 2011-2013. Rev Bras Epidemiol. 2017;20(Suppl 1):34-45

21. GBD 2017 Risk Factor Collaborators, et al. Lancet. 2018;392:1923-45 Supplementary appendix 1.

22. Vostakolaei F, Karim-Kos HE, Janssen-Heijnen ML, Visser O, Verbeek AL, Kiemeney LA. The validity of the mortality to incidence ratio as a proxy for site-specific cancer survival. Eur J Public Health. 2010;21(5):573-7.

23. Global Burden of Disease Collaborative Network. Global burden of disease study 2017 (GBD 2017) socio-demographic index (SDI) 1950-2017. Seattle, United States: Institute for Health Metrics and Evaluation (IHME), 2018. Available in: http://ghdx.healthdata.org/record/ihme-data/gbd-2017-sociodemographic-index-sdi-1950\%E2\%80\%932017. Access in: 19 Sep 2019

24. Anselin L. Local indicators of spatial association. Geographical Analysis. 1995; 27:93-115.

25. Hausman JA. Specification tests in econometrics. Econometrica. 1978;46(6): $1251-71$.

26. Pesaran $\mathrm{MH}$. Testing weak cross-sectional dependence in large panels. Econometric Reviews. 2015;34(6-10):1089-117.

27. R Core Team. R: a language and environment for statistical computing. $R$ Foundation for Statistical Computing, Vienna, Austria. [https://www.Rproject.org/]. 2017.

28. Anselin L. Spatial regression analysis in R: a workbook. 2007. [http://www. csiss.org/gispopsci/workshops/2011/PSU/readings/W15_Anselin2007.pdf] Accessed 12 July 2018.

29. Choi E, Lee S, Nhung BC, et al. Cancer mortality-to-incidence ratio as an indicator of cancer management outcomes in organization for economic cooperation and development countries. Epidemiol Health. 2017;39: e2017006.

30. Nogueira MC, Guerra MR, Cintra JRD, et al. Disparidade racial na sobrevivência em 10 anos para o câncer de mama: uma análise de mediação usando abordagem de respostas potenciais. Cad. Saúde Pública [Internet]. 2018 [citado 2019 Jan 14];34(9):e00211717.

31. Renna Junior NL, Azevedo e Silva G. Late-stage diagnosis of breast cancer in Brazil: analysis of data from hospital-based cancer registries (2000-2012). Rev Bras Ginecol Obstet. 2018;40(3):127-36.

32. da Costa AM, Hashim D, Fregnani JHTG, Weiderpass E. Overall survival and time trends in breast and cervical cancer incidence and mortality in the Regional Health District (RHD) of Barretos, São Paulo, Brazil. BMC Cancer. 2018;18(1):1079

\section{Publisher's Note}

Springer Nature remains neutral with regard to jurisdictional claims in published maps and institutional affiliations.

\section{Ready to submit your research? Choose BMC and benefit from:}

- fast, convenient online submission

- thorough peer review by experienced researchers in your field

- rapid publication on acceptance

- support for research data, including large and complex data types

- gold Open Access which fosters wider collaboration and increased citations

- maximum visibility for your research: over $100 \mathrm{M}$ website views per year

At BMC, research is always in progress.

Learn more biomedcentral.com/submissions 